\title{
Delayed-type Hypersensitivity Reaction to Glargine and Biphasic Isophane Human Insulin in a Filipino Patient with Type 2 Diabetes Mellitus: A Practical Approach to Diagnosis and Management
}

\author{
Carol Stephanie C. Tan-Lim, MD, ${ }^{1}$ Addah S. de Peralta, MDํa and Alric V. Mondragon, MD² \\ ${ }^{1}$ Division of Allergy and Immunology, Department of Pediatrics, University of the Philippines-Philippine General Hospital \\ ${ }^{2}$ Division of Allergy and Immunology, Department of Medicine, University of the Philippines-Philippine General Hospital
}

\begin{abstract}
Insulin hypersensitivity reactions are rare, but cause significant complexity in the care of patients with diabetes mellitus. A 54-year-old Filipino male with type 2 diabetes mellitus and multiple co-morbidities developed delayedtype hypersensitivity reactions to biphasic isophane human insulin and glargine. Despite good glycemic control on oral hypoglycemic agents, his endocrinologist foresaw the need for future insulin therapy, particularly one basal and one short-acting insulin. Targeted skin tests demonstrated protamine allergy and negative reactions to regular insulin and detemir. Close coordination of care among endocrinologists, allergists, patients and patients' family is needed to optimize glucose control, prevent complications, and minimize the risk of future hypersensitivity reactions.
\end{abstract}

Key Words: drug hypersensitivity, insulin, diabetes mellitus

\section{INTRODUCTION}

Since the introduction of recombinant human insulin in the 1980s, insulin hypersensitivity reactions have become rare. The current prevalence rate of insulin hypersensitivity reactions is approximately $2.4 \%$, with more than twothirds of these reactions related to the additives to the insulin product rather than to the actual insulin. ${ }^{1,2}$ Insulin hypersensitivity reactions present unique diagnostic and therapeutic challenges for clinicians due to the variety of manifestations and etiopathogenesis, the clinical complications of sudden discontinuation of insulin, and in resourcelimited settings, the lack of availability of diagnostic tests to clinch the diagnosis. This report illustrates a case of an insulin hypersensitivity reaction successfully diagnosed through simple skin tests and managed by shifting to an appropriate type of insulin.

\section{CASE REPORT}

$3^{\text {rd }}$ Place poster presentation in the Fellows' Research Forum 2019, Department of Pediatrics, at the University of the PhilippinesPhilippine General Hospital, Manila, Philippines.

Corresponding author: Carol Stephanie C. Tan-Lim, MD Division of Allergy and Immunology

Department of Pediatrics

University of the Philippines-Philippine General Hospital

Email: carolstephanietan@gmail.com

A 54-year-old Filipino male was referred to the Allergy clinic of a Philippine tertiary hospital due to insulin allergy. The patient was diagnosed with type 2 diabetes mellitus 6 years prior and was prescribed metformin. One year prior, he was admitted for ischemic stroke, and was also diagnosed with dyslipidemia and nodular toxic goiter with moderate suspicion of malignancy. $\mathrm{He}$ was started on biphasic isophane human insulin containing recombinant human 
DNA (rDNA) regular insulin and rDNA isophane insulin, atorvastatin, and carbimazole. Metformin was continued. Three months after taking these medications, the patient developed erythematous, pruritic, maculopapular rashes starting at the chest and eventually generalizing to the entire body. There were no other associated symptoms. Due to limited financial resources, the patient opted to discontinue insulin without consulting a doctor. Among the 4 drugs the patient was taking, insulin was the one discontinued since it was thought to be the most dispensable given that he was already on metformin for diabetes. The patient noted gradual resolution of rashes with the discontinuation of insulin.

Two months prior, the patient was admitted due to severe forearm trauma necessitating surgical intervention. The hospital admission was complicated due to his uncontrolled diabetes. Endocrinology service started glargine and sitagliptin in addition to metformin, and the patient was eventually discharged. The patient developed recurrence of the maculopapular rash at the chest and back after 3 weeks, prompting referral to the Allergy service. The patient opted to discontinue glargine without informing the Endocrinology service. The patient consulted at the Allergy clinic 3 days after discontinuing glargine. The rashes were already resolving, with desquamation and post-inflammatory hyperpigmentation. The patient does not have personal or family history of atopy.

The patient was assessed to have delayed-type hypersensitivity reaction to insulin. Endocrinology service was immediately informed of the discontinuation of insulin, and gliclazide was added for glucose control. Despite good glycemic control on the 3 oral hypoglycemic agents, Endocrinology service foresaw the need for future insulin administration, particularly one basal insulin and one shortacting insulin, for this patient given the complexity of his co-morbidities.

\section{Investigations}

Complete blood count, liver and kidney function tests were ordered to rule out systemic adverse effects. Results of the patient were normal.
Due to the limited funds and unavailability of specific insulin antibody testing, targeted skin testing was done, using the publication by Lee et al. as guide. ${ }^{3}$ Results of the skin test are shown in Table 1.

The patient was negative to all 3 skin prick tests. Intradermal test with regular insulin and detemir also revealed negative results within 15 minutes and after 2 days. However, intradermal test with protamine at $35 \mu \mathrm{g} / \mathrm{mL}$ concentration revealed positive results with pruritus, erythema, and an induration $>3 \times 3 \mathrm{~mm}$ compared to the negative control developing at the site of injection within 15 minutes, and persistence of induration after 2 days. From these results, the patient was diagnosed to have protamine allergy.

Based on the clinical presentation, the negative skin prick test, and the positive intradermal skin test at 15 minutes and 2 days, the patient's hypersensitivity reaction was classified as delayed-type. The positive intradermal test at 15 minutes may be due to faster reaction of delayedtype hypersensitivity upon reexposure to the antigen. An alternative explanation is that the patient may be sensitized (i.e., with $\operatorname{IgE}$ antibodies) but not have clinical manifestations of immediate-type hypersensitivity.

\section{Treatment}

Endocrinology service was advised to avoid use of protamine-containing insulin preparations, and to use regular insulin and detemir if future insulin administration is necessary. Since the patient developed rashes after use of glargine which does not contain protamine, Endocrinology service was advised to coordinate closely with Allergy service if they will restart insulin therapy, so that the patient may be carefully observed for reactions.

\section{Outcome and Follow-up}

The patient maintains good glycemic control with 3 oral hypoglycemic agents, and is on regular follow-up with Endocrinology service. Close coordination with the Endocrinology and Allergy service is maintained, which is particularly important if future insulin administration is

Table 1. Results of skin tests

\begin{tabular}{|c|c|c|c|}
\hline \multirow{2}{*}{ Agents tested } & \multirow{2}{*}{ Skin prick test } & \multicolumn{2}{|c|}{ Intradermal test } \\
\hline & & 15 minutes & 2 days \\
\hline \multicolumn{4}{|l|}{ Regular insulin } \\
\hline Undiluted (100 U/mL) & $0 \times 0 \mathrm{~mm}$ & $5 \times 5 \mathrm{~mm}$ & $0 \times 0 \mathrm{~mm}$ \\
\hline 10-fold dilution $(10 \mathrm{U} / \mathrm{mL})$ & Not done & $5 \times 5 \mathrm{~mm}$ & $0 \times 0 \mathrm{~mm}$ \\
\hline \multicolumn{4}{|l|}{ Detemir } \\
\hline Undiluted (100 U/mL) & $0 \times 0 \mathrm{~mm}$ & $5 \times 5 \mathrm{~mm}$ & $0 \times 0 \mathrm{~mm}$ \\
\hline 10-fold dilution $(10 \mathrm{U} / \mathrm{mL})$ & NA & $5 \times 5 \mathrm{~mm}$ & $0 \times 0 \mathrm{~mm}$ \\
\hline \multicolumn{4}{|l|}{ Protamine } \\
\hline Usual concentration (350 ug/mL) & $0 \times 0 \mathrm{~mm}$ & Not done & $0 \times 0 \mathrm{~mm}$ \\
\hline 10-fold dilution $(35 \mathrm{ug} / \mathrm{mL})$ & Not done & $8 \times 8 m^{*}$ & $5 \times 5 \mathrm{~mm}^{*}$ \\
\hline Histamine (positive control) & $6 \times 5 \mathrm{~mm}$ & $10 \times 9 \mathrm{~mm}$ & $0 \times 0 \mathrm{~mm}$ \\
\hline Phosphate buffered saline (negative control) & $0 \times 0 \mathrm{~mm}$ & $5 \times 5 \mathrm{~mm}$ & $0 \times 0 \mathrm{~mm}$ \\
\hline
\end{tabular}

${ }^{*}$ An induration measuring at least $3 \times 3 \mathrm{~mm}$ greater than the negative control is considered a positive result. 
necessary. If insulin administration is warranted in the future, regular insulin and detemir would be the recommended for use. If other types of insulin are needed, non-protamine containing insulin should be used. Performance of skin tests would be prudent to evaluate for potential hypersensitivity to these other types of insulin.

\section{DISCUSSION}

Insulin hypersensitivity reactions, although rare, present a unique challenge for clinicians. Based on case reports, insulin hypersensitivity reactions manifest more commonly as type I reactions such as anaphylaxis, generalized urticaria, local reactions such as pruritic, erythematous, wheals or nodules, and rarely, allergic myocardial infarction known as Kounis syndrome. ${ }^{4-11}$ Type III and type IV reactions have been reported as well. Type III reactions include Arthus type reactions, serum sickness, and leukocytoclastic vasculitis. ${ }^{12-17}$ Type IV reactions are usually reactions to additives in commercial insulin preparations and may manifest as generalized maculopapular rashes. ${ }^{18-20}$ Other manifestations with uncertain immunologic mechanisms include lipoatrophy (possibly a type III reaction), exfoliative dermatitis and gallbladder edema (possibly type IV reactions). ${ }^{21-25}$ Manifestations of insulin hypersensitivity reactions may appear after a variable period of treatment, from as early as the first insulin injection up to after 3 years of continuous insulin use. ${ }^{26-28}$ To complicate matters further, hypersensitivity reaction not just to the insulin molecule itself, but to a multitude of additives commonly found in insulin reactions have been reported. These include reactions to zinc, metacresol, and protamine sulfate..$^{24,29-31}$

Our patient manifested with maculopapular rashes 3 months after biphasic isophane human insulin, and 3 weeks after glargine use. His skin test to protamine sulfate was positive. Based on analysis of the additives in the types of insulin tested and supported by the results of the skin test, he was diagnosed to have protamine allergy (Table 2). ${ }^{12,32,33}$

Protamine is a common additive used to prolong the pharmacologic effect of insulin. It is purified from the sperm of matured testes of salmon or related fish. There are many reports of intravenous administration of protamine causing immediate reactions of anaphylaxis, urticaria, bronchospasm and hypotension due to non-immune-mediated histamine release. However, in rare cases, delayed reactions also occur. ${ }^{31}$
The results of the skin test showing protamine allergy is consistent with the clinical course of the patient reacting to the biphasic isophane human insulin, since the isophane component contains protamine. Upon skin test with just the regular insulin component, the patient did not show a reaction. However, the skin test results cannot fully explain why the patient reacted to glargine, which does not contain protamine. Possible hypersensitivity to additives found in glargine, such as zinc, metacresol and glycerol were not completely ruled out in this patient. However, skin test with detemir yielded negative result. Detemir is another longacting insulin which also contains similar additives of zinc, metacresol, and glycerol, as well as phenol. Another possibility is that the patient has hypersensitivity specific to glargine since different insulin analogues have different amino acid sequences, with studies showing that some patients may be allergic to one type of insulin but not to another type. ${ }^{6,8,20,26}$

Aside from skin tests, other tests that are commonly used to investigate insulin hypersensitivity reactions include patch test and specific $\operatorname{IgE}$ and $\operatorname{IgG}$ insulin antibody testing. Each test has its own limitations. For example, skin prick tests have poor sensitivity, with false-negative reactions occurring in 4 out of 10 patients. ${ }^{3}$ Serologic testing also has poor specificity, since studies have reported that 50 to $80 \%$ of patients on insulin therapy develop anti-insulin antibodies. ${ }^{21,31}$ Diagnostic algorithms have been published to guide clinicians in the use of these diagnostic tests. ${ }^{32,34}$

In our patient, financial constraints and limited availability of tests factored in the decision of which diagnostic tests to perform. Ultimately, the choice of diagnostic test/s to be used is affected by the clinical scenario, availability of resources, and the most updated scientific evidence. Shown in Figure 1 is the algorithm used in the management of this patient.

There are various management strategies for insulin hypersensitivity reactions, including switching to oral hypoglycemic agents, switching to a different insulin preparation, desensitization, continuing insulin therapy while giving supportive treatment, use of monoclonal antibodies, and in severe refractory cases, pancreatic transplant.

Various case reports have relayed the successful management of diabetic patients with insulin hypersensitivity with use of oral hypoglycemic agents. ${ }^{16,35,36}$ However, since type 2 diabetics generally experience gradual decline in endogenous insulin production, oral hypoglycemic agents may eventually be insufficient for glycemic control. ${ }^{34}$

Table 2. Additives in the insulin types used in the patient

\begin{tabular}{ccccc}
\hline Additives & Biphasic isophane human insulin & Glargine & Regular insulin & Detemir \\
M-cresol & + & + & + & + \\
Zinc & + & + & - & + \\
Protamine & + & - & - & - \\
Phenol & + & - & - & + \\
Glycerol & - & Maculopapular rash & Negative skin test & Negative skin test \\
\hline Patient reaction & Maculopapular rash & &
\end{tabular}


Insulin Allergy in a Filipino patient with Type 2 Diabetes Mellitus

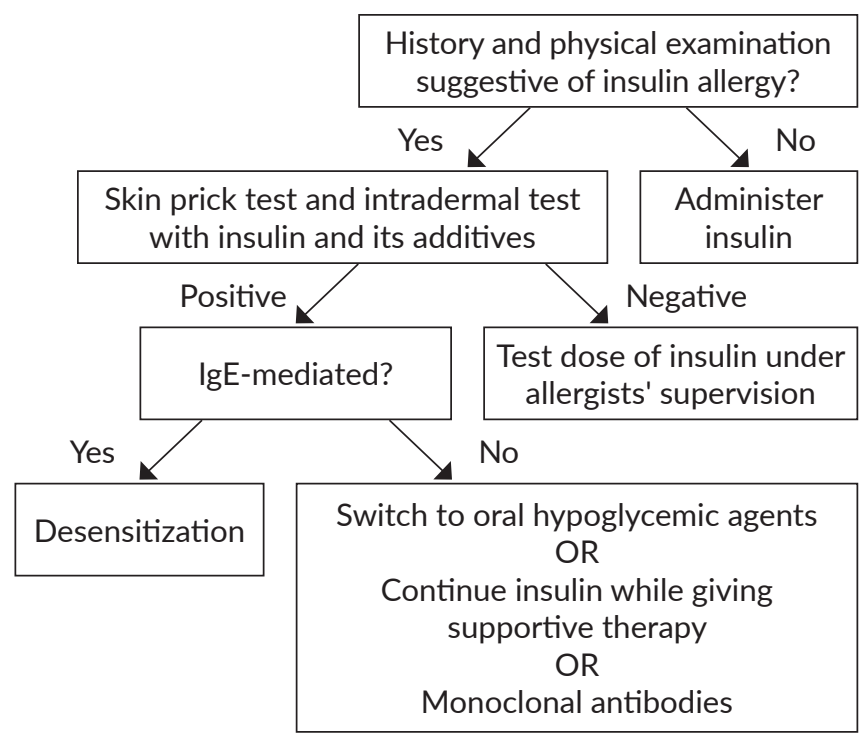

Figure 1. Algorithm used in the management of this patient.

Other case reports noted successful switching to a different insulin preparation, especially in patients that have hypersensitivity reactions to additives in the insulin preparation. ${ }^{29}$ It is curious to note however, that success has been reported even in patients without documented hypersensitivity to additives. ${ }^{6,8,20,26,37}$ These reports do not have a common insulin type that is considered less likely to cause hypersensitivity reactions, as each patient reacted to a wide variety of insulin types and was successfully switched to different types of insulin. The differences in amino acid sequences among the different insulin types have been proposed to be one of the reasons for this success. ${ }^{32}$ However, this should be done carefully and with adequate precautions, due to the risk of anaphylaxis. ${ }^{27}$

Desensitization using a variety of desensitization protocols, including the use of continuous subcutaneous insulin infusion, have also been conducted successfully among patients with insulin hypersensitivity. ${ }^{4,5,12,18,38,39}$ Other less commonly reported strategies include administration of immunosuppressants while continuing insulin therapy, use of omalizumab, and pancreatic transplant. ${ }^{17,19,40}$

Our patient was successfully managed with switching to oral hypoglycemic agents. However, due to the complicated medical history, his endocrinologist foresaw the need for insulin therapy in the future. Thus, targeted skin prick tests were done using the most commonly used and easily accessible insulin preparations in the local market to identify a different type of insulin that the patient would have less likelihood of having a hypersensitivity reaction to.

\section{CONCLUSION}

This case report illustrates the unique challenge of diagnosing and treating patients with insulin hypersensitivity reactions, particularly in resource-limited settings. Close coordination with endocrinologists and allergists is necessary to ensure good glycemic control while minimizing the risk of hypersensitivity reactions. Careful analysis of the patient history, the additives used in common insulin preparations, and targeted skin testing can help clinicians choose the most appropriate treatment for these patients.

\section{Statement of Authorship}

All authors participated in data collection and analysis, and approved the final version submitted

\section{Author Disclosure}

All authors declared no conflicts of interest

\section{Funding Source}

The authors did not receive funding for this paper.

\section{REFERENCES}

1. Rojas J, Villalobos M, Martinez MS, Chavez-Castillo, M, Torres W, Mejias JC, et al. Successful management of insulin allergy and autoimmune polyendocrine syndrome type 4 with desensitization therapy glucocorticoid treatment: a case report and review of the literature. Case Reports Immunol 2014;2014:394754.

2. Ghazavi MK and Johnston GA. Insulin allergy. Clinics in Dermatology 2011;29:300-5.

3. Lee AY, Chey WY, Choi J, Jeon JS. Insulin-induced drug eruptions and reliability of skin tests. Acta Derm Venereol 2002;82:114-7.

4. Luyasu S, Hougardy N, Hasdenteufel F, Jacquenet S, Weber E, Moneret-Vautrin A, et al. Anaphylactic shock due to recombinant human insulin: follow-up of a desensitization protocol by basophil activation test. Rev Med Interne. 2011;32(1):39-42.

5. Hasani-Ranjbar S, Fazlollahi MR, Mehri A, Larijani B. Allergy to human insulin and specific immunotherapy with glargine; case report with review of literature. Acta Diabetol 2012;49:69-73.

6. Adachi A, Fukunaga A, and Horikawa T. A case of human insulin allergy induced by short-acting and intermediate-acting insulin but not by long-acting insulin. Int J Dermatol 2004;43(8):597-9.

7. Durand-Gonzalez KN, Guillausseau N, Pecquet C, Gayno JP. Glargine insulin is not an alternative in insulin allergy. Diabetes Care 2003;26(7):2216.

8. Sokup A, Swiatkowski M, Tyloch M, Szymanski W. Insulin Lispro as an alternative for insulin Humulin $\mathrm{U}$ in the treatment of an obese gestational diabetic woman with allergy to Humulin U. Case report. Pregl Lek 2005;62(4):260-1.

9. Andrade P, Barros L and Goncalo M. Type $1 \mathrm{IgE}$ mediated allergy to human insulin, insulin analogues and beta-lactam antibiotics. An Bras Dermatol 2012;87(6):917-9.

10. Fujikawa T, Imbe H, Date M, Go Y, Kitaoka H. Severe insulin allergy successfully treated with continuous subcutaneous insulin infusion. Diabetes Research and Clinical Practice 2012;97:e31-3.

11. Ozlek B, Ozlek E, Celik O, Cil C, Dogan V, Biteker M. Allergic myocardial infarction following recombinant human insulin. Heart Lung 2018147(4):360-2.

12. Pfohler C, Muller CSL, Hasselmann DO, Tilgen W. Successful desensitization with human insulin in a patient with an insulin allergy and hypersensitivity to protamine: a case report. Journal of Medical Case Reports 2008;2:283.

13. Aujero MP, Brooks S, Li N, Venna S. Severe serum sickness-like type III reaction to insulin detemir. J Am Acad Dermatol 2011;64(6): e127-8.

14. Bayraktar F, Akinci B, Dermikan F, Yener S, Yesil S, Kirmaz C, et al. Serum sickness-like reactions associated with type III insulin allergy responding to plasmapheresis. Diabetic Medicine 2009;26:659-60. 
15. Marusic S, Vlahovic-Palcevski V and Ljubanovic D. Leukocytoclastic vasculitis associated with insulin aspart in a patient with type 2 diabetes. Int J Clinical Pharmacol Ther 2009;47(10):603-5.

16. Rachid B, Rabelo-Santos M, Mansour E, de Lima Zollner R, Velloso LA. Type III hypersensitivity to insulin leading to leukocytoclastic vasculitis. Diabetes Research and Clinical Practice 2010;89:e39-40.

17. Murray BR, Jewell JR, Jackson KJ, Agboola O, Alexander BR, Sharma P. Type III hypersensitivity reaction to subcutaneous insulin preparations in a type 1 diabetic. J Gen Intern Med 2017;32(7):841-5.

18. Moyes V, Driver R, Croom A, Mirakian R, Chowdhury TA. Insulin allergy in a patient with type 2 diabetes successfully treated with continuous subcutaneous insulin infusion. Diabetic Medicine 2006;23:204-6.

19. Koroscil T, Kagzi $Y$ and Zacharias D. Failure of multiple therapies in the treatment of a type 1 diabetic patient with insulin allergy: a case report. Endocrine Practice 2011;17(1):91-4.

20. Alfadhli E. Allergy to insulin glargine: a case report. J Med Cases 2011;2(1):4-6.

21. Tavare AN, Doolittle HJ and Baburaj R. Pan-insulin allergy and severe lipoatrophy complicating type 2 diabetes. Diabetic Med 2011;28(4):500-3.

22. Cabrera-Freitag P, Escalada J, Goikoetxea MJ, Laguna S, Sanz ML, Gastaminza G. A severe case of lipoatrophy due to human insulin and insulin analogs in a patient with diabetes: is an immunological mechanism involved? J Investig Allergol Clin Immunol 2011; 21(5):410-21.

23. Beltrand J, Guilmin-Crepon S, Castanet M,Peuchmaur M, Czernichow P, Levy-Marchal C. Insulin allergy and extensive lipoatrophy in child with type 1 diabetes. Horm Res 2006;65:253-60.

24. Rajpar SF, Foulds IS, Abdullah A, Maheshwari M. Severe adverse cutaneous reaction to insulin due to cresol sensitivity. Contact Dermatitis 2006;55:119-20.

25. Kawasaki F, Kamei S, Tatsumi F, Hamomoto S, Shimoda M, Tawaramoto K, et al. Gallbladder edema in type 1 diabetic patient due to delayed-type insulin allergy. Inter Med 2009;48:1545-9.

26. Perez E, Gonzalez R, Martinez J, et al. Detemir insulin-induced anaphylaxis. Ann Allergy Asthma Immunol. 2009;102(2):174-5.

27. Kaya A, Gungor K and Karakose S. Severe anaphylactic reaction to human insulin in a diabetic patient. Journal of Diabetes and Its Complications. 2007;21:124-7.

28. Hirai H, Ogata E, Kikuchi N, Kohno T, Machii N, Hasegawa K, et al. The effects of liraglutide on both hypereosinophilic insulin allergy and the characteristics of anti-insulin antibodies in type 2 diabetes mellitus: a case report. Journal of Medical Case Reports 2016;10:202.
29. Ammar IB, Ksouri H, Trabelsi N, Mellouli F, Mami FB, Dakhli S, et al. Generalized allergy due to zinc in insulin treated with zinc-free insulin. Acta Diabetol 2012;49:239-41.

30. Clerx V, Van Den Keybus C, Kochuyt A, et al. Drug intolerance reaction to insulin therapy caused by metacresol. Contact Dermatitis 2003;48(3):162-3.

31. Raap U, Liekenbrocker T, Kapp A, Wedi B. Delayed-type hypersensitivity to protamine as a complication of insulin therapy. Contact Dermatitis 2005;53:57-8.

32. Heinzerling L, Raile K, Rochlitz H, Zuberbier T, Worm M. Insulin allergy: clinical manifestations and management strategies. Allergy 2008;63:148-55.

33. Humulin 70/30. [Internet] [cited 2019 March 21]. Available from: https://www.rxlist.com/humulin-70-30-drug.htm.

34. Haastrup MB, Henriksen JE, Mortz CG, Bindslev-Jensen C. Insulin allergy can be successfully managed by a systematic approach. Clin Transl Allergy 2018;8:35.

35. Jialal I and Singh R. Oral pharmacotherapy as alternative treatment for type 2 diabetes mellitus in a 61 year old ethnic Filipino man with insulin allergy. Laboratory Medicine 2019;50(1):93-5.

36. Takata H, Kumon Y, Osaki F, Kumagai C, Arii K, Ikeda Y, et al. The human insulin analogue aspart is not the almighty solution for insulin allergy. Diabetes Care 2003;26(1):253-4.

37. Fujishiro M, Izumida Y, Takemiya S, Kuwano Y, Takamoto I, Suzuki R, et al. A case of insulin allergy successfully managed using multihexamer-forming insulin degludec combined with liraglutide. Diabet Med 2016;33(11):e26-9.

38. Egufluz-Gracia I, Rodriguez-Alvarez M, Cimarra-Alvarez M, Sanabria-Perez, MC, Martinez-Cocera C. Desensitization for insulin allergy: a useful treatment also for local forms. J Investig Allergol Clin Immunol 2012;22(3):215-35.

39. Hara M, Izumida Y, Sato N, Ohashi K, Osuga JI, Tobe K, et al. Successful desensitization by glargine administration in a patient with insulin allergy: a case report. Diabetes Research and Clinical Practice 2009;84:e48-9.

40. Cavelti-Weder C, Muggli B, Keller C, Babians-Brunner A, BiasonLauber A, Donath M, et al. Successful use of omalizumab in an inadequately controlled type 2 diabetic patient with severe insulin allergy. Diabetes Care 2012;35(6):e41. 